\title{
Carbonic anhydrase 2 and 3 as risk biomarkers for dilated cardiomyopathy associated heart failure
}

\author{
Hao Su^, Kai Hu, Zhiquan Liu, Kangyu Chen, Jian Xu \\ Department of Cardiology, The First Affiliated Hospital of University of Science and Technology of China (Anhui Provincial Hospital), Hefei, China \\ Contributions: (I) Conception and design: H Su; (II) Administrative support: K Chen, Jian Xu; (III) Provision of study materials or patients: K Hu, Z \\ Liu, K Chen, J Xu; (IV) Collection and assembly of data: H Su, K Hu, Z Liu; (V) Data analysis and interpretation: H Su, K Chen; (VI) Manuscript \\ writing: All authors; (VII) Final approval of manuscript: All authors. \\ Correspondence to: Hao Su, MD. Department of Cardiology, The First Affiliated Hospital of University of Science and Technology of China (Anhui \\ Provincial Hospital), Lujiang Road 17, Hefei, China. Email: suhaod@126.com.
}

\begin{abstract}
Background Dilated cardiomyopathy (DCM) is a complex type of cardiomyopathy that is affected by both genetic and non-genetic factors. It is characterized by an enlargement of the left ventricle or bi-ventricle, and is often accompanied by cardiac systolic dysfunction. The main results include arrhythmia, heart failure (HF), and sudden death. The prognosis of this disease is usually poor, and the 5 -year survival time is about $50 \%$. Early diagnosis is very important for the treatment of DCM. Studies have shown that primary prevention after discovering the disease effectively reduces the mortality rate of the disease. However, there is currently no effective biomarker for the early diagnosis of DCM. The rapid development of omics in protein has promoted the "precise" study of modern medical research. In this article, the potential biomarkers for predicting and diagnosing DCM-related HF were studied by a plasma protein omics analysis.
\end{abstract}

Methods: Tandem mass tag-labeled quantitative proteomic studies were performed in 20 patients, comprising 10 DCM-associated HF patients, and 10 control patients who without clinical HF events. Further validation research was conducted by enzyme-linked immunosorbent assay (ELISA) with an expanded cohort (control group $=40 ;$ HF group $=48$ ).

Results: Among the 854 identified proteins, the expression of 86 proteins was significantly upregulated, while the expression of 21 proteins was downregulated (with an expression difference $>1.5$-fold; $\mathrm{P}<0.05$ ) in the 2 groups. The Gene Ontology, Kyoto Encyclopedia of Genes and Genomes pathway enrichment, and protein-protein interaction (PPI) networks analyses indicated that the bicarbonate transport process played a critical role in HF. Importantly, carbonic anhydrase 2 (CA2) and 3 (CA3), which play central roles in regulating the transport of bicarbonate, were highly expressed in the HF group. The ELISA validation results showed that the expression levels of CA2 and CA3 at admission were remarkably higher $(\mathrm{P}<0.0001$ and $\mathrm{P}=0.0157$ ) in the plasma of the HF patients than that of the control patients.

Conclusions: The present study showed that two molecules (i.e., CA2 and CA3) are involved in the bicarbonate transport pathway, and are risk factors and potential biomarkers for the diagnosis of DCM patients with $\mathrm{HF}$.

Keywords: Dilated cardiomyopathy (DCM); heart failure (HF); proteomics; biomarkers; carbonic anhydrase

Submitted Oct 25, 2021. Accepted for publication Dec 16, 2021.

doi: 10.21037/apm-21-3561

View this article at: https://dx.doi.org/10.21037/apm-21-3561

\footnotetext{
^ ORCID: 0000-0002-0061-0066.
} 


\section{Introduction}

Dilated cardiomyopathy (DCM) is a non-ischemic heart muscle disease with structural and functional myocardial abnormalities, characterized by left ventricular or biventricular dilation, and impaired contraction that cannot be explained by abnormal loading conditions or coronary artery disease (1). The etiologies of DCM are complicated, and mainly include genetic mutations, infections, inflammation, autoimmune diseases, toxin exposure, and metabolic and endocrine dysfunction (2). Currently, symptomatic treatment is the key method for preventing serious complications in DCM patients. However, in the case of unknown causes, the question of how to treat clinical symptoms, such as arrhythmia and heart failure (HF), is challenging for cardiologists and emergency physicians.

Chronic HF is a serious clinical syndrome occurring in the decompensation stage of $\operatorname{DCM}(3,4)$. Many patients with DCM may progress to chronic HF, which is frequently accompanied by other organ failure, such as kidney and lung failure, and a poor prognosis $(3,4)$. Among DCM patients (hospitalized and ambulatory), most sudden deaths are due to worsening HF. The latest European data from the EURObservational Research Programme: The Heart Failure Pilot Survey shows that the 12-month all-cause mortality rates of hospitalized and stable and ambulatory HF patients were $17 \%$ and $7 \%$, respectively, while the $12-$ month hospitalization rates of those patients were $44 \%$ and $32 \%$, respectively $(5,6)$. Thus, the pre-onset characteristics and risk factors or sensitive biomarkers of HF need to be identified urgently. Traditionally, N-terminal proB-type natriuretic peptide (NT-proBNP) is a broadspectrum serological marker for the evaluation of the risk of $\mathrm{HF}$ in various cardiovascular diseases $(7,8)$. However, with advancements in genomic, proteomic, and metabolic profiling technology, modern medicine has gradually been moving in the direction of personalized "precision" medicine (9-11). This will not only help us to understand the detailed process of the development of certain subtypes of diseases, but will also provide new blood-based biomarkers that will further improve our ability to stratify risks and customize treatments according to patient' unique molecular characteristics.

Cardiomyopathy is divided into ischemic cardiomyopathy and non-ischemic cardiomyopathy, while DCM is one of the non-ischemic diseases. CA1 and CA2 are overexpressed in diabetic ischemic myocardium. In this article, we report on the discovery of 2 promising biomarkers for the prediction of DCM-associated HF; that is, carbonic anhydrase 2 (CA2) and 3 (CA3). Pathological cardiac hypertrophy, the maladaptive remodelling of the myocardium, often progresses to heart failure. The sodium-proton exchanger (NHE1) and chloride-bicarbonate exchanger (AE3) have been implicated as important in the hypertrophic cascade. CA2 physically interacts with NHE1 and AE3, enhancing their respective ion transport activities by increasing the concentration of substrate at their transport sites. CA3 is a cytosolic isoform of the $\alpha$-CA family which catalyze the physiological reaction of carbon dioxide $\left(\mathrm{CO}_{2}\right)$ hydration to bicarbonate and proton, which also has been proposed to play a new role in cells where oxidative damage occurs. Tandem mass tag (TMT)-labeling-based quantitative proteomics was used to assess $\mathrm{HF}$-associated proteomic changes in the plasma of DCM patients. Additionally, Gene Ontology (GO) functional enrichment, Kyoto Encyclopedia of Genes and Genomes (KEGG) pathway enrichment, and protein-protein interaction (PPI) bioinformatics analyses were performed on the differentially expressed proteins to evaluate the biological functions most closely related to the changed proteins. We found that CA2 and CA3 were significantly upregulated in the HF group and were enriched in the bicarbonate transport pathway. Both molecules may be used as risk factors and represent the most promising biomarkers for the prediction and diagnosis of HF in DCM patients. We present the following article in accordance with the MDAR reporting checklist (available at https://dx.doi.org/10.21037/apm-21-3561).

\section{Methods}

\section{Study design and patient enrollment}

The experiment process fully complied with the Clinical Experiment Ethics Code of The First Affiliated Hospital, University of Science and Technology, China. We recruited 108 patients to participate in this study. In brief, the patients were divided into 2 groups, the control group (comprising 50 patients) and the HF group (comprising 58 patients), based on the corresponding indicator. Patients in the control group usually had a medical history of arrhythmias, including atrioventricular nodal reentrant tachycardia, atrioventricular nodal dual pathways, premature ventricular contraction, and sick sinus syndrome. Notably, these patients had no significant organic heart changes and their left ventricular ejection fraction (LVEF) was generally $\geq 50 \%$. Patients in the HF group were diagnosed 
with DCM combined with LVEF $\leq 40 \%$ and NT-proBNP $\geq 2,000 \mathrm{pg} / \mathrm{mL}$, had an obviously enlarged heart (as observed in X-ray and echocardiography examinations), and had the main symptom of congestive $\mathrm{HF}$ accompanied by various complex arrhythmias. The plasma used for the TMT-labeled protein mass spectrometry (MS) analysis was collected randomly from 10 patients in the control and HF groups, respectively, and the rest was used for subsequent enzymelinked immunosorbent assay (ELISA) verification. The characteristics and statistical information of the 20 patients involved in the MS are set out in Table 1. The study was conducted in accordance with the Declaration of Helsinki (as revised in 2013). The study was approved by the ethics committee of Anhui Provincial Hospital (No. 2016-163). All the patients signed the informed consent form.

\section{Plasma collection and storage}

The venous blood of all the patients was collected before any clinical intervention treatment commenced during the hospitalization, and stored at room temperature in vacutainer ethylenediaminetetraacetic acid tubes to prevent hemolysis. Plasma, obtained after centrifugation at 2,000 g for $15 \mathrm{~min}$, was transferred into small aliquots, and stored at $-80{ }^{\circ} \mathrm{C}$ for further analysis (12).

\section{Proteomic protocols and bioinformatics analysis}

First, the Pierce ${ }^{\mathrm{TM}}$ Top 12 Abundant Protein Depletion Spin Columns Kit (Thermo Fisher; Waltham, USA) was used to remove the top 12 high abundance proteins from the plasma samples. Next, the protein concentration was measured by a bicinchoninic acid kit according to the manufacturer's instructions. For digestion, the protein solution was alkylated with $11 \mathrm{mM}$ of iodoacetamide for $15 \mathrm{~min}$ at room temperature in darkness, and was then reduced with $5 \mathrm{mM}$ of dithiothreitol at $56{ }^{\circ} \mathrm{C}$ for $30 \mathrm{~min}$. Next, trypsin was added twice for digestion at a 1:50 and 1:100 trypsinto-protein mass ratio with a urea concentration $<2 \mathrm{M}$. Trypsin digested peptides were then desalted by the Strata X C18 SPE column (Phenomenex; Torrance, USA), vacuum-dried, reconstituted in $0.5 \mathrm{M}$ triethylammonium bicarbonate (TEAB), and processed according to the manufacturer's protocol for the TMT kit. Finally, the peptides were subjected to a nano ESI-MS (NSI) source followed by tandem MS (MS/MS) in Q Exactive ${ }^{\mathrm{TM}}$ Plus (Thermo; Waltham, USA) coupled online to the ultra- performance liquid chromatography (UPLC).

The resulting MS/MS data were processed using the Maxquant search engine (v.1.5.2.8). Tandem mass spectra were searched against the human uniprot database concatenated with the reverse decoy database. The GO annotation proteome was derived from the UniProt-GOA database (http://www.ebi.ac.uk/GOA/), and InterProScan soft (v.5.14-53.0) was used to annotate the protein's domain functional description based on the protein sequence alignment method. Differentially expressed proteins were identified by $\mathrm{P}$ values $<0.05$ and $\log _{2}$ fold change $\geq 1.5$ or $\leq 0.67$.

\section{ELISA}

The contents of the CA2 and CA3 in the plasma were measured by human CA2 and CA3 ELISA kits (CUSABIO; CSB-E08823h, CSB-E15962h; Wuhan, China) in accordance with the manufacturer's standard protocol. The fluorescence intensity of each well was then measured by an 800 TS absorbance reader (BioTek; Winooski, USA). Finally, the concentration was calculated in accordance with the manufacturer's instructions.

\section{Statistical analysis}

Statistical analyses were performed using Prism (v.8.0.1, GraphPad Software). For all the statistical analyses, the values were compared using Student's $t$-tests for the two group comparisons. ${ }^{*} \mathrm{P}<0.05 ;{ }^{* *} \mathrm{P}<0.01 ;{ }^{* *} \mathrm{P}<0.001$, and the data are shown as the mean \pm standard error of the mean.

\section{Results}

\section{Patient cohort}

All patients (58 HF patients and 50 control patients) enrolled in this study were from The First Affiliated Hospital, University of Science and Technology, China. Among them, 20 contributors whose plasma was used for the TMT-labeled protein MS were selected randomly and their patient characteristics and statistical information are summarized in Table 1. The HF patients in this study were all clinically diagnosed with DCM. The HF group of patients was characterized by a history of cardiac insufficiency diagnosed before the present hospitalization and had a history of long-term use of antihypertensive drugs, such as angiotensin-converting enzyme inhibitor 
Table 1 Baseline features and statistical information of patients in the HF group and the control group

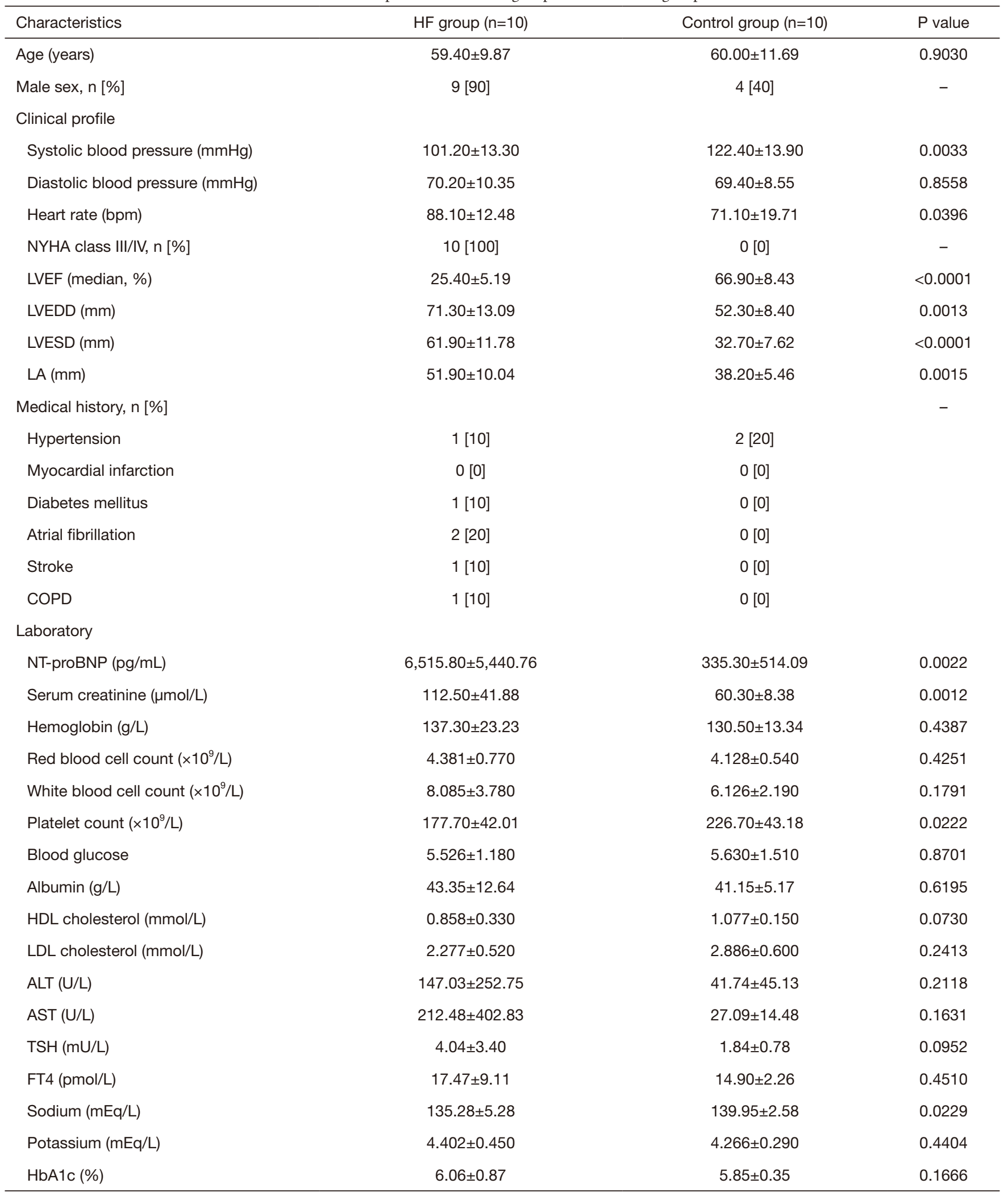

Table 1 (continued) 
Table 1 (continued)

\begin{tabular}{lcc}
\hline Characteristics & HF group $(\mathrm{n}=10)$ & Control group $(\mathrm{n}=10)$ \\
\hline Medication, $\mathrm{n}[\%]$ & & $\mathrm{P}$ value \\
ACE/ARB & $3[30]$ & $1[10]$ \\
Beta-blocker & $6[60]$ & $1[10]$ \\
Aldosterone antagonist & $9[90]$ & $0[0]$ \\
Loop diuretic & $7[70]$ & $0[0]$ \\
Digoxin & $3[30]$ & $0[0]$ \\
\hline
\end{tabular}

Data are expressed as mean \pm standard deviation if not otherwise specified. HF, heart failure; NYHA, New York Heart Association; LVEF, left ventricular ejection fraction; LVEDD, left ventricular end-diastolic dimension; LVESD, left ventricular end-systolic dimension; LA, left atrium; COPD, chronic obstructive pulmonary disease; NT-proBNP, NT-proB-type natriuretic peptide; HDL, high-density lipoprotein; ALT, alanine aminotransferase; AST, aspartate aminotransferase; TSH, thyroid stimulating hormone; FT4, free thyroxine; ACE, angiotensinconverting enzyme; ARB, angiotensin receptor blocker.

(ACEI)/angiotensin-receptor blocker (ARB), beta blockers, aldosterone receptor antagonist, diuretics, and digoxin, which drugs rarely used in the control group. Notably, the HF patients presented with organic heart disease and were in a decompensated period, but the patients in both groups were similar in terms of age. Specifically, compared to the control group, a higher New York Heart Association (NYHA) class of III/IV (30\% and 70\%) and a lower LVEF $(<40 \%, 25.40 \% \pm 5.19 \%)$ indicated that the symptoms of HF had occurred. Additionally, the echocardiographic results showed that the left ventricular end-diastolic dimension (LVEDD) $(71.30 \pm 13.09$ vs. $52.30 \pm 8.40 \mathrm{~mm} ; \mathrm{P}=0.0013)$, left ventricular end-systolic dimension (LVESD) $(61.90 \pm 11.78$ vs. $32.70 \pm 7.62 \mathrm{~mm} ; \mathrm{P}<0.0001)$ and left atrium (LA) (51.90 \pm 10.04 vs. $38.20 \pm 5.46 \mathrm{~mm} ; \mathrm{P}=0.0015)$ dimension were all higher in the HF group than the control group. NT-proBNP, which is the most commonly used biomarker for the diagnosis of HF, was about 20 times higher in the HF group than control group $(6,515.80 \pm 5,440.76 v s$. $335.30 \pm 514.09 \mathrm{pg} / \mathrm{mL} ; \mathrm{P}=0.0022)$. In addition, while the serum creatinine level in the HF group was within the normal range, it was significantly higher than that of the control group, which suggests that impaired renal function might be associated with HF. Clinically, cardiorenal syndrome is a common complication of advanced HF, and renal insufficiency is an independent risk factor for the death of patients with acute HF (13).

\section{Quantitative proteomics and an overview of the identified proteins}

Figure $1 A$ summarizes the overall design of this study. In the first stage, 11,414 unique peptide fragments and 994 corresponding proteins were identified in the TMT-labeled protein quantitative MS experiment (see Figure 1B). Three statistical analysis methods, including a principal component analysis, relative standard deviation, and Pearson's correlation coefficient, were used to assess the repeatability of the quantitative protein (see Figure $1 C$ and Figure S1A). Among them, 854 proteins were quantified and constituted the raw data for subsequent bioinformatics analysis (see Figure $1 B$ ) (the raw data are available via ProteomeXchange. project ID: IPX0001802000). In addition, compared to the control group, the expression levels of the 107 proteins, including 86 upregulated and 21 downregulated proteins, in the HF group had a significantly greater change ( $>1.5$ fold; $\mathrm{P}<0.05$; see Figure 1D).

\section{Bioinformatics analysis of the significant differentially expressed proteins}

The GO enrichment analysis revealed that the molecular functions were primarily related to binding, catalytic activity, and structural activity, while the biological processes mainly involved biological regulation, cellular processes, and single-organism processes (see Figure S1A). Based on the Wolfpsort and CELLO software analysis, the subcellular localization distribution of the 107 significant differentially expressed proteins involved extracellular (44.86\%), cytoplasm $(28.04 \%)$, nucleus $(12.15 \%)$, plasma membrane $(6.54 \%)$, mitochondria $(5.61 \%)$, and other (2.8\%) (see Figure S1B). Next, we conducted further GO classification and KEGG pathway enrichment analyses (the $P$ values were obtained using the Fisher's exact test) for the 
A Clinical examination

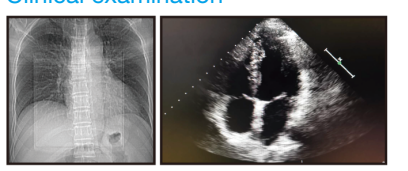

X-ray

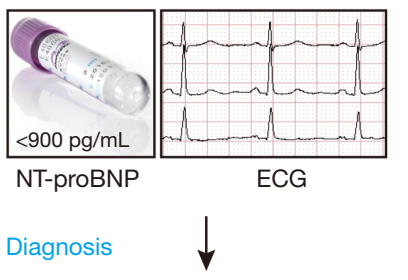

AVNRT/AVNDP

PVC/SSS

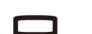

을

Venous blood collection (anticoagulant)

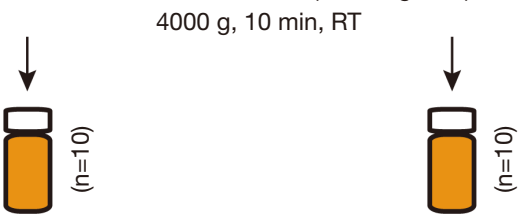

Plasma collection $\left(-80^{\circ} \mathrm{C}\right)$

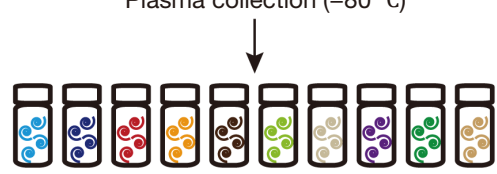

Protein mass spectrometry analysis (TMT labeled quantitative)

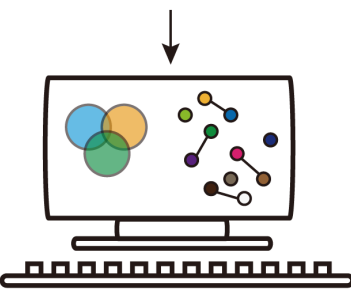

Bioinformatics analysis of proteomics data

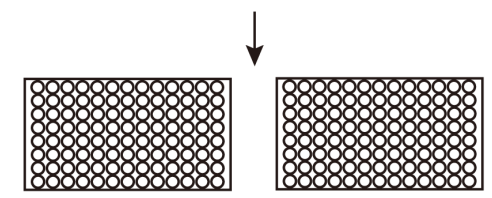

Candidate biomarkers prediction and verification

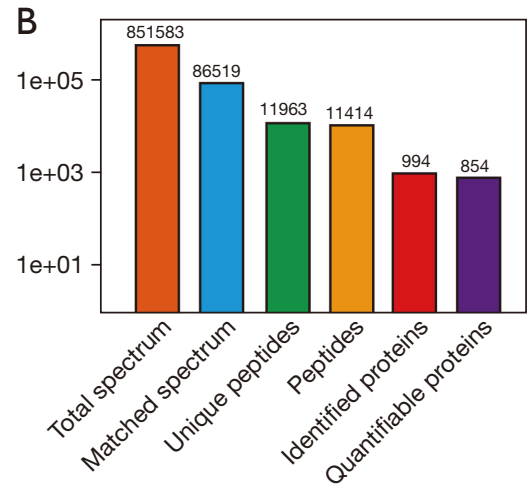

C

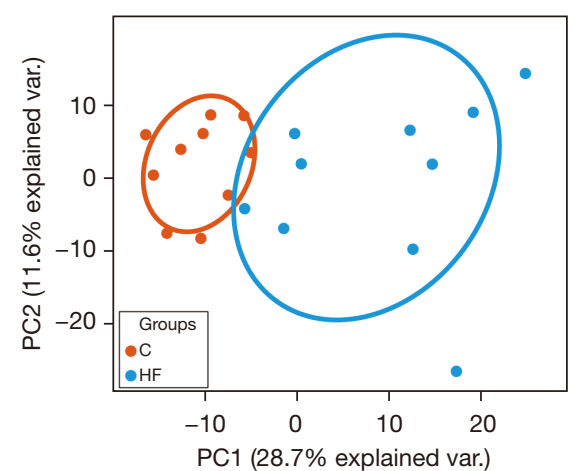

D

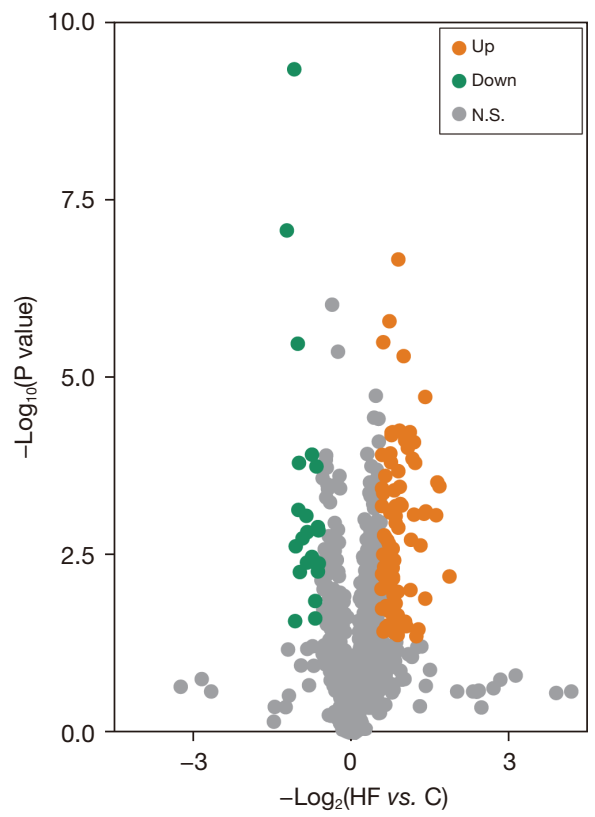

Figure 1 Study workflow and overview of the proteomic results. (A) Study workflow; (B) quantities of peptides and protein identified by TMT-labeled protein quantitative MS; (C) principal component analysis score plot; (D) volcano map showing differentially expressed proteins between the control and HF groups $(\mathrm{P}<0.05)$. NT-proBNP, NT-proB-type natriuretic peptide; ECG, electrocardiogram; AVNRT, Atrioventricular nodal reentry tachycardia; AVNDP, Dual atrioventricular nodal pathways; PVC, premature ventricular contraction; SSS, Sick sinus syndrome; DCM, dilated cardiomyopathy; RT, room temperature; TMT, tandem mass tag; MS, mass spectrometry; HF, heart failure. 
upregulated and downregulated proteins. As Figure $2 A, 2 B$ show, the 86 upregulated proteins were mainly involved in the molecular functions of carbonate dehydratase activity, antioxidant activity, and mothers against decapentaplegic homolog (SMAD) binding, simultaneously, and participated in biological processes, such as cellular oxidant detoxification and protein complex assembly. Conversely, the 21 downregulated proteins were mainly involved in the molecular functions of nucleoside-triphosphatase activity, pyrophosphatase activity, and hydrolase activity, and participated in biological processes, such as responses to oxygen-glucose deprivation, mitochondrion morphogenesis, and cellular responses to oxygen-glucose deprivation (see Figure 2C,2D). Additionally, the KEGG pathway enrichment analysis showed that those upregulated proteins were overrepresented in nitrogen metabolism (hsa00910), platelet activation (hsa04611), the advanced glycation end-products (AGE)-receptor for AGE (RAGE) signaling pathway in diabetic complications (hsa04933), and collecting duct acid secretion (hsa04966) (see Figure 2E). The downregulated protein was enriched in the herpes simplex virus 1 infection (hsa05168), phototransduction (hsa04744), and choline metabolism in cancer (hsa05231) pathway (see Figure 2F).

\section{Biomarkers predict DCM patients with HF}

Among the 107 proteins, the top 15 upregulated proteins and the top 15 downregulated proteins are listed in Figure $3 A, 3 B$. A PPI analysis of these 30 proteins was conducted at https://string-db.org (see Figure $3 C, 3 D$ ). Notably, consistent with previous results (see Figure 2A), CA2, solute carrier family 4 member 1 (SLC4A1), and hemoglobin subunit alpha 1 (HBA1) are closely related (see Figure 3C), and were significantly enriched in the biological process of bicarbonate transport combined with CA3 [GO:0015701, false discovery rate (FDR) 4.74e-05] (see tables available at https://cdn.amegroups.cn/static/ public/apm-21-3561-1.xlsx). Bicarbonate has been shown to play a critical role in cardiac cells benefiting from the regulation of intracellular $\mathrm{pH}\left(\mathrm{pH}_{\mathrm{i}}\right)(14,15) \cdot \mathrm{pH}_{\mathrm{i}}$ is an important modulator of cardiac excitation and contraction, and a potent trigger of electrical arrhythmia (16). The balance of $\mathrm{pH}_{\mathrm{i}}$ is broken in patients with DCM because of the ischemia and hypoxia of heart combined with metabolic acidosis (17), which suggests that the bicarbonate transport process could be monitored to reflect heart burden and predict the risk of HF.

\section{Validation of CA2 and CA3 by ELISA experiment in vitro}

Combined with the bioinformatics results in Figure 2 and previous findings, $\mathrm{CA} 2$ and $\mathrm{CA} 3$ are the most promising risk biomarkers for DCM-associated HF (18-23). Next, we used the plasma of 40 control patients and 48 DCM patients with HF symptoms for further ELISA experiment validation. Consistent with the results of the MS, the CA2 and CA3 levels in the HF group were significantly higher than those in the control group [CA2: $1.70(0.00-5.80) v s$. $3.64(0.36-15.34) \mathrm{ng} / \mathrm{mL}, \mathrm{P}<0.0001$; CA3: $27.26(0.00$ $211.24)$ vs. $62.73(0.00-405.69) \mathrm{ng} / \mathrm{mL}, \mathrm{P}=0.0157]$ (see Figure $3 E, 3 F)$. These encouraging results suggest that CA2 and CA3 may serve as risk biomarkers for DCM of HF and warrant further studies.

\section{Discussion}

DCM is a chronic heart disease and once it develops into HF, patient prognosis is often poor (24). Thus, it is imperative to find a specific and sensitive serological marker to monitor the risk of HF in DCM patients. We identified a total of 854 quantified proteins and 107 significantly differentially expressed proteins, including 86 upregulated and 21 downregulated proteins (more than 1.5 -fold at $\mathrm{P}<0.05)$. These results suggest that the pathophysiological process of HF is complicated and related to a wide range of differential protein expressions.

Subsequently, the bioinformatics analyses showed that carbonate dehydratase activity (see Figure $2 A$, related protein: CA1, CA2, CA3) and nitrogen metabolism (see Figure 2E, related protein: CA1, CA2, CA3) were considered the most significant molecular function in those distinctively upregulated proteins. Additionally, the PPI analysis of the top 15 upregulated proteins indicated that CA2, SLC4A1, and HBA1 are closely related to and enriched in the biological process of bicarbonate transport combined with CA3. Notably, CA1, CA2, and CA3 are all members of the carbonic anhydrase (CA) family that are responsible for maintaining the acid-base balance and helping $\mathrm{CO}_{2}$ transportation. CAs are ubiquitous metalloenzymes, present throughout most living organisms and encoded by six unrelated gene families: the $\alpha-, \beta-, \gamma-, \delta-, \eta-$, and $\zeta$-Cas (25). CAs catalyze a very simple reaction: the hydration of $\mathrm{CO}_{2}$ to bicarbonate and proton. The elevation of CA1, CA2, and $\mathrm{CA} 3$ levels may indicate the disturbance of acid-base balance and disorder of bicarbonate or $\mathrm{CO}_{2}$ transportation in $\mathrm{HF}$-associated DCM patients. We further validated 
A

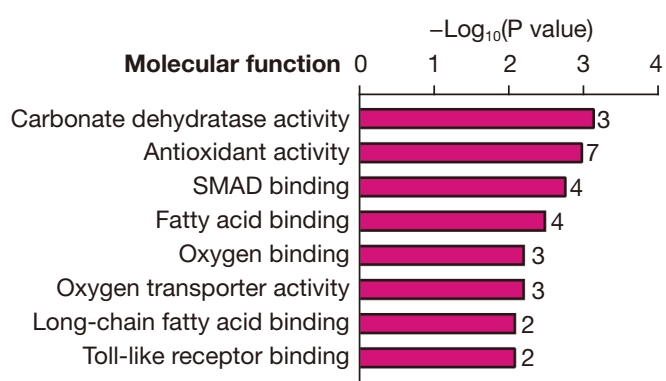

C
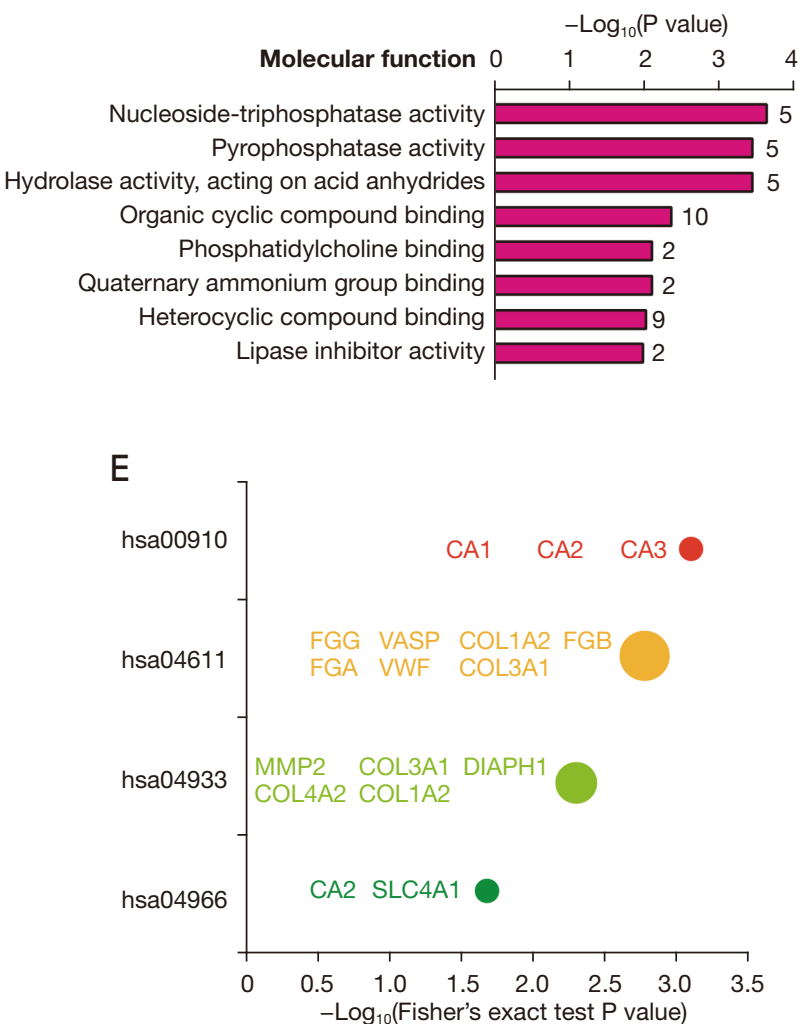

B

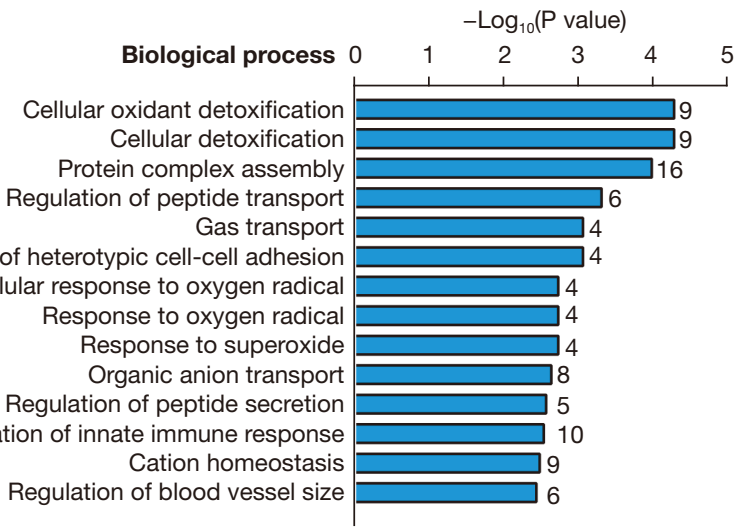

D
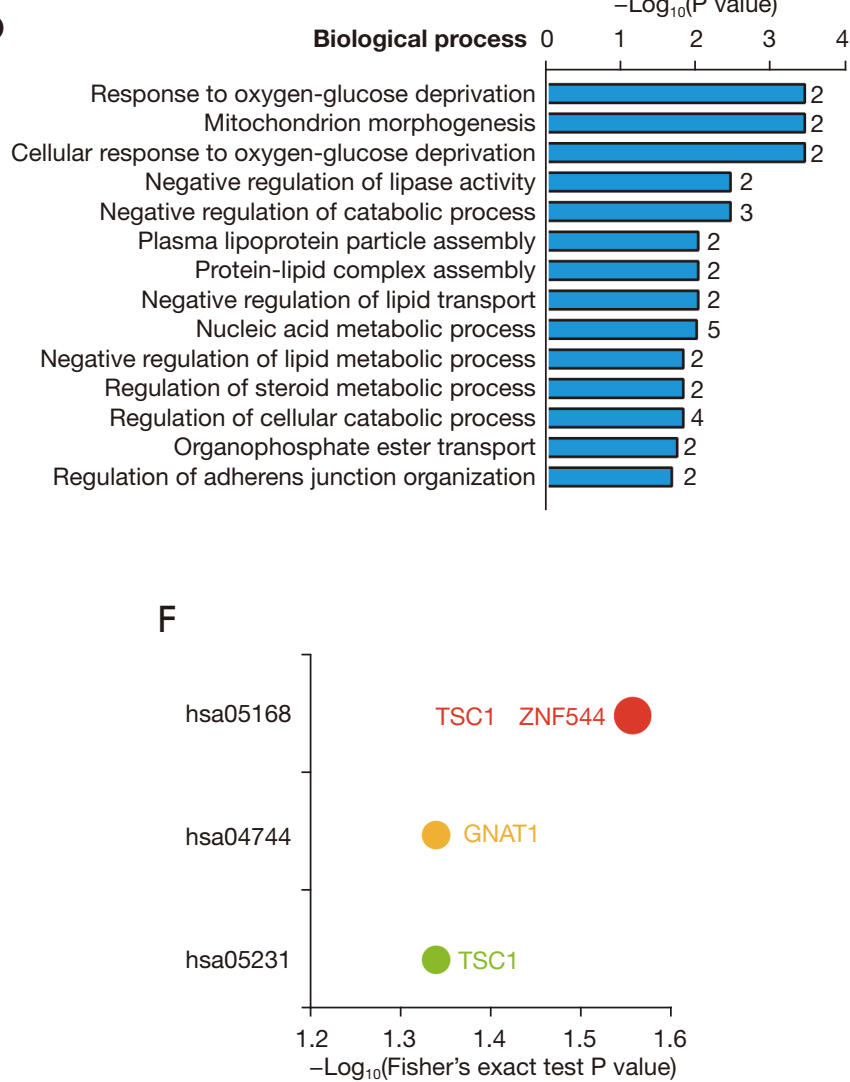

Figure 2 GO classification and KEGG pathway enrichment analyses for significantly differentially expressed proteins. (A) GO classification of 86 upregulated proteins, and classification of proteins based on molecular function; (B) GO classification of 86 upregulated proteins, and classification of proteins based on biological process; (C) GO classification of 21 downregulated proteins, and classification of proteins based on molecular function; (D) GO classification of 21 downregulated proteins, and classification of proteins based on biological process; (E) KEGG pathway enrichment analysis of upregulated proteins; (F) KEGG pathway enrichment analysis of downregulated proteins. CA, carbonic anhydrase; GO, Gene Ontology; KEGG, Kyoto Encyclopedia of Genes and Genomes.

these candidate molecules by an ELISA experiment, and evaluated the reliability of the CA family as new biomarkers of DCM-associated HF.
$\mathrm{pH}_{\mathrm{i}}$ is an important modulator of cardiac excitation and contraction, and also a potent trigger of electrical arrhythmia. $\mathrm{H}^{+}$-equivalent transporters, such as $\mathrm{Na}^{+}-\mathrm{H}^{+}$ 
A

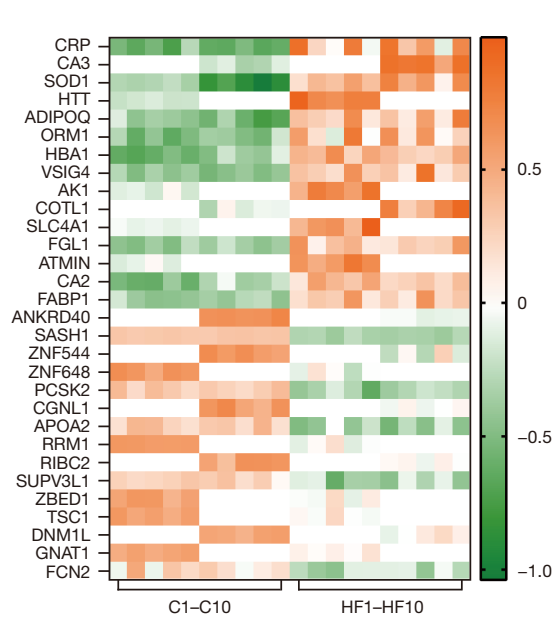

C

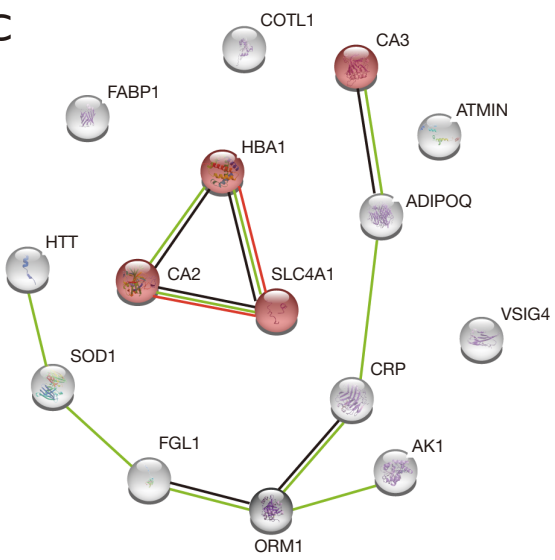

E

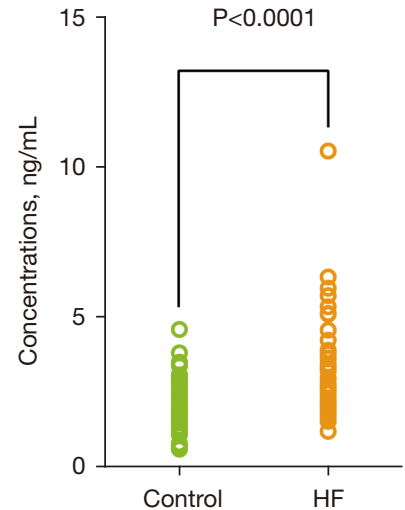

B

\begin{tabular}{lccc}
\hline Gene & Fold change & Expression & $P$-value \\
\hline CRP & 3.658 & Up & $6.24 \mathrm{E}-03$ \\
CA3 & 3.199 & Up & $3.35 \mathrm{E}-04$ \\
SOD1 & 3.119 & Up & $2.96 \mathrm{E}-04$ \\
HTT & 3.077 & Up & $8.61 \mathrm{E}-04$ \\
ADIPOQ & 2.681 & Up & $7.61 \mathrm{E}-04$ \\
ORM1 & 2.651 & Up & $1.28 \mathrm{E}-02$ \\
HBA1 & 2.649 & Up & $1.86 \mathrm{E}-05$ \\
VSIG4 & 2.628 & Up & $2.29 \mathrm{E}-04$ \\
AK1 & 2.496 & U 1 & $3.48 \mathrm{E}-02$ \\
COTL1 & 2.436 & Up & $4.32 \mathrm{E}-02$ \\
SLC4A1 & 2.364 & Up & $1.57 \mathrm{E}-04$ \\
FGL1 & 2.326 & Up & $8.42 \mathrm{E}-04$ \\
ATMIN & 2.294 & Up & $8.05 \mathrm{E}-04$ \\
CA2 & 2.291 & Up & $1.38 \mathrm{E}-04$ \\
FABP1 & 2.248 & Down & $8.44 \mathrm{E}-08$ \\
ANKRD40 & 0.428 & $4.54 \mathrm{E}-10$ \\
SASH1 & 0.474 & Down & $2.65 \mathrm{E}-02$ \\
ZNF544 & 0.480 & Down & $2.36 \mathrm{E}-03$ \\
ZNF648 & 0.484 & Down & $3.29 \mathrm{E}-06$ \\
PCSK2 & 0.496 & Down & $7.23 \mathrm{E}-04$ \\
CGNL1 & 0.501 & Down & $1.57 \mathrm{E}-04$ \\
APOA2 & 0.502 & Down & $5.42 \mathrm{E}-03$ \\
RRM1 & 0.509 & DNM & $1.82 \mathrm{E}-03$ \\
RIBC2 & 0.527 & Down & $8.77 \mathrm{E}-04$ \\
SUPV3L1 & 0.556 & Down & $3.98 \mathrm{E}-03$ \\
ZBED1 & 0.560 & Down & $1.48 \mathrm{E}-03$ \\
TSC1 & 0.563 & Down & $3.32 \mathrm{E}-03$ \\
DNM1L & 0.597 & Down & $1.20 \mathrm{E}-04$ \\
GNAT1 & 0.598 & Down & $2.43 \mathrm{E}-02$ \\
FCN2 & 0.623 & & \\
\hline & &
\end{tabular}

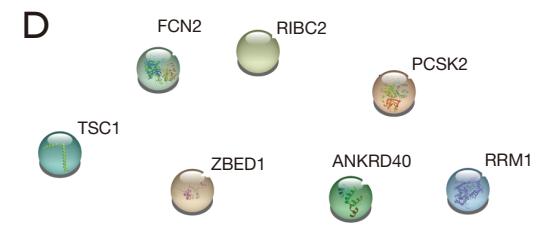

(2) GNAT

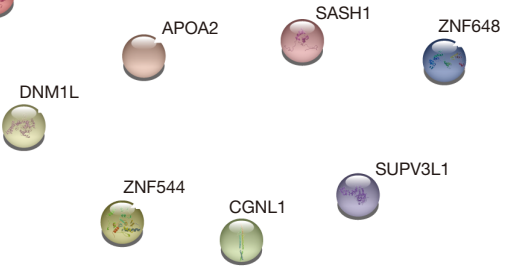

$\mathrm{F}$

CA3

$P=0.0157$

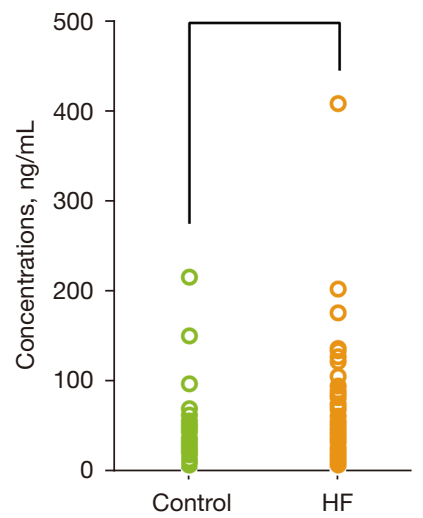

Figure 3 CA2 and CA3 may serve as risk biomarkers for DCM of HF. (A) The folding change levels of the top 15 raised proteins and the top 15 lowered proteins as indicated by orange (increase) and green (decrease), respectively; (B) detailed data of the fold change of the top 15 upregulated proteins and the top 15 downregulated proteins; (C) PPI analysis of the top 15 upregulated proteins; (D) PPI analysis of the top 15 downregulated proteins; (E) ELISA test detects CA2 levels in DCM patients with HF symptoms and control patients $(\mathrm{P}<0.0001$; paired Student's $t$-test); (F) ELISA test detects CA3 level in DCM patients with HF symptoms and control patients ( $\mathrm{P}=0.0157$; paired Student's $t$-test). CA, carbonic anhydrase; DCM, dilated cardiomyopathy; HF, heart failure; PPI, protein-protein interaction. 
exchanger (NHE), sodium-bicarbonate cotransporters (NBC), chloride-bicarbonate exchangers (CBE), $\mathrm{Cl}^{-}$$\mathrm{OH}^{-}$exchanger (CHE) and monocarboxylate transporters (MCT), have all been shown to be involved in the $\mathrm{pH}$ regulation of cardiac myocytes. The CA family can spatially regulate the $\mathrm{pH}_{\mathrm{i}}$ by catalyzing the interconversion between $\mathrm{CO}_{2}$ and water and controlling the dissipation of metabolic $\mathrm{CO}_{2}$ (26,27). In addition, the CA family has been shown to enhance the $\mathrm{pH}$ regulation ability of $\mathrm{H}^{+}$-equivalent transporters by binding to the carrier protein. The higher expression of CA2 and CA4 was confirmed in hypertrophic/ dilated ventricles consistent with atrial natriuretic peptide (ANP) and brain natriuretic peptide (BNP) in messenger ribonucleic acid and protein levels (20). Some studies have found that a broad-spectrum CA inhibitor can prevent the process of cardiomyocyte hypertrophy $(\mathrm{CH})$, which suggests that CA plays a critical role in the development of $\mathrm{CH}$ (22). It has also been reported that CA exists in the blood in soluble form (28). Consistent with our MS and ELISA experiments, these results robustly demonstrated that CA2 and $\mathrm{CA} 3$ are promising biomarkers of DCM-associated HF and that sequential CA2 and CA3 measurements reflect the clinical course of DCM combined with NT-proBNP. NT-proBNP is a broad-spectrum serological marker for the evaluation of the risk of HF (7). However, CA2 and CA3 will provide new blood-based biomarkers that will further improve our ability to stratify risks and customize treatments according to patient' unique molecular characteristics.

This study had some limitations. First, the plasma of 10 typical clinical patients for each group was collected for the TMT-labeled quantitative proteomic studies; however, the sample size was relatively small for each group. Further ELISA verification for the extended samples is necessary. In future research, we will examine changes in the CA family during the development of DCM and their correlations with prognosis. Second, 107 significant differentially expressed proteins were identified to predict the occurrence of DCMassociated HF. In this biomarker pool, while CA2 and $\mathrm{CA} 3$ are considered the 2 most promising risk biomarkers, other candidate biomarkers may play a synergistic role in improving the predictive power of CA2 and CA3 for DCMassociated HF, and should be further investigated. Third, previous studies reported that CA2 and CA3 are associated with gastrointestinal tumors (29), Alzheimer's disease (28), thyroid disease (30), and neuromuscular diseases (31). In this study, anhydrase 2 and 3 were confirmed to use as risk biomarkers for dilated cardiomyopathy associated heart failure, however, the mechanism of significant increase of the CA in non-ischemic heart muscle disease in not clear, which still needs further research. Thus, the specificity of $\mathrm{CA} 2$ and $\mathrm{CA} 3$ as new markers requires further exploration.

\section{Acknowledgments}

Funding: None.

\section{Footnote}

Reporting Checklist: The authors have completed the MDAR reporting checklist. Available at https://dx.doi. org/10.21037/apm-21-3561

Data Sharing Statement: Available at https://dx.doi. org/10.21037/apm-21-3561

Conflicts of Interest: All authors have completed the ICMJE uniform disclosure form (available at https://dx.doi. org/10.21037/apm-21-3561). The authors have no conflicts of interest to declare.

Ethical Statement: The authors are accountable for all aspects of the work in ensuring that questions related to the accuracy or integrity of any part of the work are appropriately investigated and resolved. The study was conducted in accordance with the Declaration of Helsinki (as revised in 2013). The study was approved by the ethics committee of Anhui Provincial Hospital (No. 2016-163). All the patients signed the informed consent form.

Open Access Statement: This is an Open Access article distributed in accordance with the Creative Commons Attribution-NonCommercial-NoDerivs 4.0 International License (CC BY-NC-ND 4.0), which permits the noncommercial replication and distribution of the article with the strict proviso that no changes or edits are made and the original work is properly cited (including links to both the formal publication through the relevant DOI and the license). See: https://creativecommons.org/licenses/by-nc-nd/4.0/.

\section{References}

1. Schultheiss HP, Fairweather D, Caforio ALP, et al. Dilated cardiomyopathy. Nat Rev Dis Primers 2019;5:32.

2. McKenna WJ, Maron BJ, Thiene G. Classification, Epidemiology, and Global Burden of Cardiomyopathies. 
Circ Res 2017;121:722-30.

3. Ponikowski P, Voors AA, Anker SD, et al. 2016 ESC Guidelines for the diagnosis and treatment of acute and chronic heart failure: The Task Force for the diagnosis and treatment of acute and chronic heart failure of the European Society of Cardiology (ESC). Developed with the special contribution of the Heart Failure Association (HFA) of the ESC. Eur J Heart Fail 2016;18:891-975.

4. Seferović PM, Polovina M, Bauersachs J, et al. Heart failure in cardiomyopathies: a position paper from the Heart Failure Association of the European Society of Cardiology. Eur J Heart Fail 2019;21:553-76.

5. Maggioni AP, Dahlström U, Filippatos G, et al. EURObservational Research Programme: regional differences and 1-year follow-up results of the Heart Failure Pilot Survey (ESC-HF Pilot). Eur J Heart Fail 2013;15:808-17.

6. Bui AL, Horwich TB, Fonarow GC. Epidemiology and risk profile of heart failure. Nat Rev Cardiol 2011;8:30-41.

7. Chow SL, Maisel AS, Anand I, et al. Role of Biomarkers for the Prevention, Assessment, and Management of Heart Failure: A Scientific Statement From the American Heart Association. Circulation 2017;135:e1054-91.

8. Daniels LB, Maisel AS. Natriuretic peptides. J Am Coll Cardiol 2007;50:2357-68.

9. Leopold JA, Loscalzo J. Emerging Role of Precision Medicine in Cardiovascular Disease. Circ Res 2018;122:1302-15.

10. Cresci S, Pereira NL, Ahmad F, et al. Heart Failure in the Era of Precision Medicine: A Scientific Statement From the American Heart Association. Circ Genom Precis Med 2019;12:458-85.

11. Ibrahim NE, Januzzi JL Jr. Established and Emerging Roles of Biomarkers in Heart Failure. Circ Res 2018;123:614-29.

12. Malmström E, Kilsgård $\mathrm{O}$, Hauri S, et al. Large-scale inference of protein tissue origin in gram-positive sepsis plasma using quantitative targeted proteomics. Nat Commun 2016;7:10261.

13. Núñez J, Miñana G, Santas E, et al. Cardiorenal Syndrome in Acute Heart Failure: Revisiting Paradigms. Rev Esp Cardiol (Engl Ed) 2015;68:426-35.

14. Wang HS, Chen Y, Vairamani K, et al. Critical role of bicarbonate and bicarbonate transporters in cardiac function. World J Biol Chem 2014;5:334-45.

15. Vaughan-Jones RD, Spitzer KW. Role of bicarbonate in the regulation of intracellular $\mathrm{pH}$ in the mammalian ventricular myocyte. Biochem Cell Biol 2002;80:579-96.
16. Vaughan-Jones RD, Spitzer KW, Swietach P. Intracellular $\mathrm{pH}$ regulation in heart. J Mol Cell Cardiol 2009;46:318-31.

17. Urso C, Brucculeri S, Caimi G. Acid-base and electrolyte abnormalities in heart failure: pathophysiology and implications. Heart Fail Rev 2015;20:493-503.

18. Vaughan-Jones RD, Villafuerte FC, Swietach P, et al. pHRegulated $\mathrm{Na}(+)$ influx into the mammalian ventricular myocyte: the relative role of $\mathrm{Na}(+)-\mathrm{H}(+)$ exchange and $\mathrm{Na}(+)-\mathrm{HCO}$ Co-transport. J Cardiovasc Electrophysiol 2006;17 Suppl 1:S134-40.

19. Villafuerte FC, Swietach P, Youm JB, et al. Facilitation by intracellular carbonic anhydrase of $\mathrm{Na}+-\mathrm{HCO} 3-$ co-transport but not $\mathrm{Na}+/ \mathrm{H}+$ exchange activity in the mammalian ventricular myocyte. J Physiol 2014;592:991-1007.

20. Alvarez BV, Quon AL, Mullen J, et al. Quantification of carbonic anhydrase gene expression in ventricle of hypertrophic and failing human heart. BMC Cardiovasc Disord 2013;13:2.

21. Torella D, Ellison GM, Torella M, et al. Carbonic anhydrase activation is associated with worsened pathological remodeling in human ischemic diabetic cardiomyopathy. J Am Heart Assoc 2014;3:e000434.

22. Alvarez BV, Johnson DE, Sowah D, et al. Carbonic anhydrase inhibition prevents and reverts cardiomyocyte hypertrophy. J Physiol 2007;579:127-45.

23. Cingolani HE, Ennis IL. Sodium-hydrogen exchanger, cardiac overload, and myocardial hypertrophy. Circulation 2007;115:1090-100.

24. McNally EM, Mestroni L. Dilated Cardiomyopathy: Genetic Determinants and Mechanisms. Circ Res 2017;121:731-48

25. Monti DM, De Simone G, Langella E, et al. Insights into the role of reactive sulfhydryl groups of Carbonic Anhydrase III and VII during oxidative damage. J Enzyme Inhib Med Chem 2017;32:5-12.

26. Supuran CT, Scozzafava A. Carbonic anhydrases as targets for medicinal chemistry. Bioorg Med Chem 2007;15:4336-50.

27. Arthurs GJ, Sudhakar M. Carbon dioxide transport. Continuing Education in Anaesthesia Critical Care \& Pain 2005;5:207-10.

28. Jang BG, Yun SM, Ahn K, et al. Plasma carbonic anhydrase II protein is elevated in Alzheimer's disease. J Alzheimers Dis 2010;21:939-45.

29. Parkkila S, Lasota J, Fletcher JA, et al. Carbonic anhydrase II. A novel biomarker for gastrointestinal stromal tumors. 
Mod Pathol 2010;23:743-50.

30. Auton JA, Barragry JM, Carter ND, et al. Rapid diagnosis of thyroid disease using carbonic-anhydrase immunoassay. Lancet 1976;2:1385-7.

31. Heath R, Schwartz MS, Brown IR, et al. Carbonic

Cite this article as: $\mathrm{Su} \mathrm{H}, \mathrm{Hu} \mathrm{K}$, Liu Z, Chen $\mathrm{K}, \mathrm{Xu} \mathrm{J}$. Carbonic anhydrase 2 and 3 as risk biomarkers for dilated cardiomyopathy associated heart failure. Ann Palliat Med 2021;10(12):12554-12565. doi: 10.21037/apm-21-3561 anhydrase III in neuromuscular disorders. J Neurol Sci 1983;59:383-8.

(English Language Editor: L. Huleatt) 


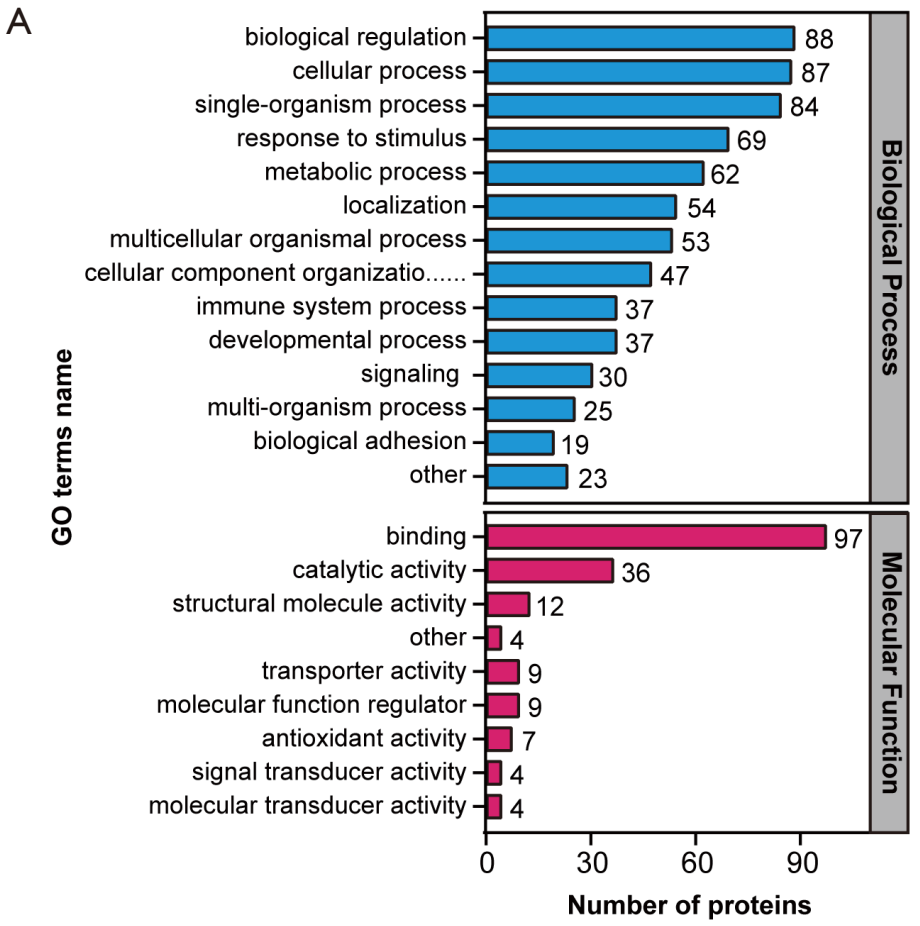

B

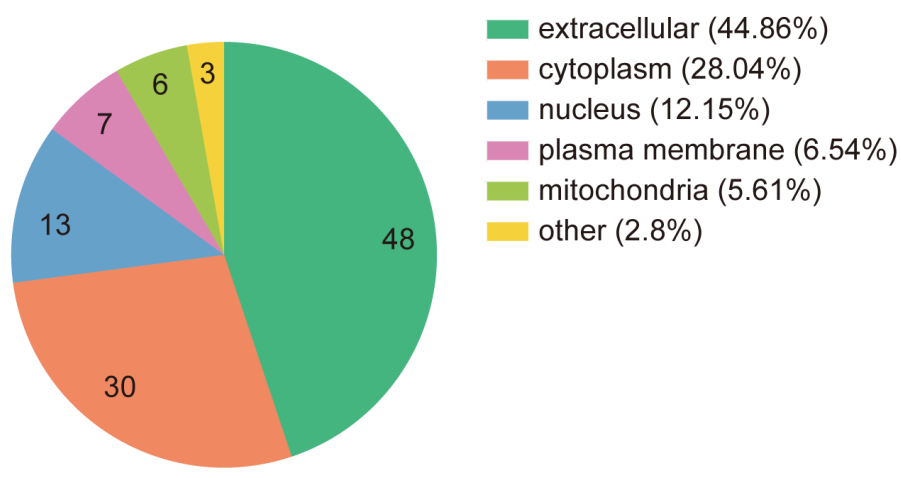

Figure S1 Bioinformatics analysis of significant differentially expressed proteins. (A) GO enrichment analysis of the significantly differentially expressed proteins, and classification of proteins according to their biological process and molecular function; (B) 107 subcellular locations of differentially expressed proteins were calculated using Wolf sort and CELLO software. GO, Gene Ontology. 